\title{
Alternated Julia sets and connectivity properties
}

\author{
Marius-F. Danca ${ }^{1}$, M. Romera ${ }^{2}$, G. Pastor $^{2}$ \\ ${ }^{1}$ Department of Applied Sciences, Avram Iancu University, 3400 Cluj-Napoca, Romania \\ ${ }^{2}$ Instituto de Física Aplicada, Consejo Superior de Investigaciones Científicas, Serrano 144, 28006 Madrid, Spain
}

In this paper we present the alternated Julia sets, obtained by alternated iteration of two maps of the quadratic family $z_{n+1}=z_{n}^{2}+c_{i}, i=1,2$ and prove analytically and computationally that these sets can be connected, disconnected or totally disconnected verifying the known Fatou-Julia theorem in the case of polynomials of degree greater than two. A few examples are presented.

Keywords: Julia set; Quadratic polynomials; Connectivity; Fatou-Julia theorem

\section{Introduction}

The evolution of a natural process can be modeled by using discrete dynamical systems, that is to say, maps which apply one point to another point of certain variables space. Note that in Nature there are many different interactions and therefore systems do not evolve according to a unique dynamics. Therefore, it is reasonable to think that the evolution of a natural process should be explained by the alternated iteration of different dynamics. Let us assume, to simplify, that we have only two different discrete dynamics $D_{1}$ and $D_{2}$, corresponding to seasonal variations in the model of the natural process. In this case we can study the out-coming dynamics $D$ by combination of the dynamics $D_{1}$ and $D_{2}$, i.e., $D: x_{0} \stackrel{D_{1}}{\longrightarrow} x_{1} \stackrel{D_{2}}{\longrightarrow} x_{2} \stackrel{D_{1}}{\longrightarrow} x_{3} \ldots$, where $\left\{x_{0}, x_{1}, x_{2}, \ldots\right\}$ are the values of the variable $x$ describing the physical system [1].

In this paper we will study the alternated iteration of pairs of maps of the complex quadratic family $z_{n+1}=z_{n}^{2}+c_{i}, i=1,2$ by means of its Julia sets. The alternated iteration of two real logistics maps was firstly studied in [2] using computer tools as histograms and surrogate tests. Moreover, the synthesis of hyperbolic attractors of 
continuous dynamical systems by switching the control parameter, in a regular deterministic manner, was obtained in [3].

As is known, the filled Julia set of a complex polynomial $P: \mathbb{C} \rightarrow \mathbb{C}$ is $K=\left\{z \in \mathbb{C} \mid P^{\circ n}(z) \rightarrow \infty\right\}$ where $P^{\circ n}$ denotes the $n^{\text {th }}$ iteration of $P$. The Julia set of $P$ is $J=\partial K$, i.e. what you get when you remove the inside of the filled Julia set, leaving only its boundary part. As is also known, $J$ is connected if and only if $K$ is connected and, therefore, $J$ and $K$ have the same connectivity properties.

As is also known, the connectivity properties of the Julia set for a polynomial of degree $d \geq 2$ have an intimate relationship with the dynamical properties of its finite critical points. In 1918-1919 Fatou [4] and Julia [5] proved a result what is known as the Fatou-Julia theorem:

(i) The Julia set is connected if and only if all the critical orbits are bounded.

(ii) The Julia set is totally disconnected, a Cantor set ${ }^{1}$, if (but not only if) all the critical orbits are unbounded.

In 1992, Branner and Hubbard [7] stated a conjecture that was proved by Qiu and Yin in 2006 [8]. Hence it completes the Fatou-Julia theorem as follows:

(iii) For a polynomial with at least one critical orbit unbounded, the Julia set is totally disconnected if and only if all the bounded critical orbits are aperiodic.

The parts (i) and (ii) of the theorem treat the two extreme cases where either all the critical points or none of the critical points have bounded orbits. In between [9-11], if one or more critical orbits are unbounded and one or more critical orbits are bounded, the Julia set is either disconnected or totally disconnected according to part (iii). A disconnected Julia set consists of infinitely many pieces, some of which may be points, but others are connected sets that are not points.

We briefly recall a well known result for the quadratic polynomial $P_{c}(z)=z^{2}+c$. Each map $P_{c}$ has a single critical point at 0 and a single critical orbit.

\footnotetext{
${ }^{1}$ A Cantor set is defined as a compact, perfect, totally disconnected subset in $\mathbb{C}$. Any such set is homeomorphic to the middle-third Cantor set and therefore deserves the name a Cantor set. Since any Julia set is compact and perfect it follows that a given Julia set is a Cantor set if and only if it is totally disconnected [6].
} 
The fate of this orbit leads to the fundamental dichotomy for quadratic polynomials [12, 13] that is the Fatou-Julia theorem for degree $d=2$ :

(i) The Julia set of $P_{c}$ is connected if the orbit of 0 is bounded.

(ii) The Julia set of $P_{c}$ is totally disconnected if the orbit of 0 is unbounded.

The set of parameter values $c$ for which the Julia set of $P_{c}$ is connected forms the well known Mandelbrot set.

Even though the Julia set of a map of the quadratic family $z_{n+1}=z_{n}^{2}+c$ can be either connected or totally disconnected, in this paper we will show that the Julia set of the alternated iteration of two maps of the quadratic family can be connected, totally disconnected and also disconnected.

\section{Alternated Julia sets}

Let us consider the family of quadratic maps $P_{c}: z_{n+1}=z_{n}^{2}+c$. We introduce the alternated filled Julia set $K_{c_{1} c_{2}}$ as being the set of points of the complex plane whose orbits are bounded when we iterate the alternated system

$$
P_{c_{1} c_{2}}: z_{n+1}=\left\{\begin{array}{l}
z_{n}^{2}+c_{1}, \text { if } n \text { is even } \\
z_{n}^{2}+c_{2}, \text { if } n \text { is odd }
\end{array}\right.
$$

and, in the same manner, we introduce the alternated filled Julia set $K_{c_{2} c_{1}}$ as being the set of points of the complex plane whose orbits are bounded when we iterate the alternated system

$$
P_{c_{2} c_{1}}: z_{n+1}=\left\{\begin{array}{l}
z_{n}^{2}+c_{2}, \text { if } n \text { is even } \\
z_{n}^{2}+c_{1}, \text { if } n \text { is odd. }
\end{array}\right.
$$

The odd and even iterates of $P_{c_{1} c_{2}}$ respond to the general expressions

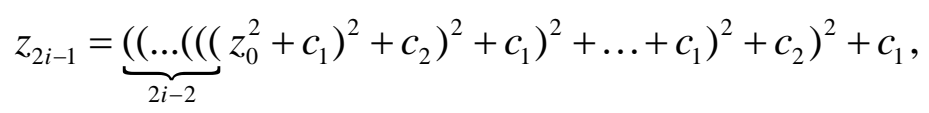


and

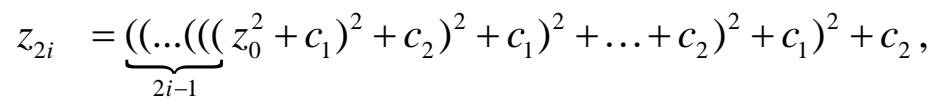

where $z_{0}$ is the initial value and $i=1,2,3 \ldots$ We have $z_{2 i}=z_{2 i-1}^{2}+c_{2}$ and $z_{2 i+1}=z_{2 i}^{2}+c_{1}$.

\section{Proposition 2.1}

(i) If $z_{2 i}$ is bounded, $z_{2 i-1}$ is also bounded.

(ii) If $z_{2 i}$ is unbounded, $z_{2 i+1}$ is also unbounded.

PROOF. If $z_{2 i}$ is bounded it is obvious that $z_{2 i-1}=\sqrt{z_{2 i}-c_{2}}$ is also bounded. If $z_{2 i}$ is unbounded it is obvious that $z_{2 i+1}=z_{2 i}^{2}+c_{1}$ is also unbounded.

Because the study of the connectivity of the Julia sets of $P_{c_{1} c_{2}}$ is a difficult task, we introduce the auxiliary complex quartic polynomial $Q_{c_{1} c_{2}}$

$$
Q_{c_{1} c_{2}}: z_{n+1}^{*}=\left(z_{n}^{* 2}+c_{1}\right)^{2}+c_{2}
$$

The iterates of $Q_{c_{1} c_{2}}$ respond to the general expression

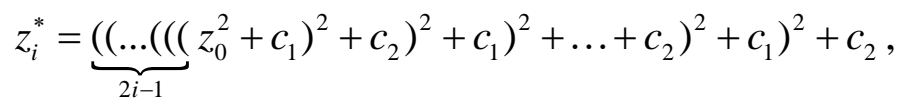

where $z_{0}$ is the initial value and $i=1,2,3 \ldots$

\section{Theorem 2.1}

The (un)boundedness of $\left\{Q_{c_{1} c_{2}}^{\circ n}\right\}$ implies the (un)boundedness of $\left\{P_{c_{1} c_{2}}^{\circ n}\right\}$.

ProOf. Equations (2. 4) and (2. 6) show that $z_{2 i}=z_{i}^{*}$. Let's next consider the orbits of $P_{c_{1} c_{2}}$ and $Q_{c_{1} c_{2}}$ for the same initial value $z_{0}$. Taking into account proposition 2.1, if the orbit of $Q_{c_{1} c_{2}}$ is bounded the orbit of $P_{c_{1} c_{2}}$ is also bounded; if the orbit of $Q_{c_{1} c_{2}}$ is unbounded, the orbit of $P_{c_{1} c_{2}}$ is also unbounded. 
Therefore we can enunciate the following main theorem

\section{Theorem 2.2}

The Julia set of the alternated system $P_{c_{1} c_{2}}$ and the Julia set of the quartic system $Q_{c_{1} c_{2}}$ are the same for given $c_{1}$ and $c_{2}$ parameter values.

\section{Examples}

Figure 1 shows the Julia set of the quadratic map $z_{n+1}=z_{n}^{2}+(-0.76+0.1 i)$. As is well known, this Julia set is totally disconnected because $-0.76+0.1 i$ does not belong to the Mandelbrot set. However, the alternated Julia sets $K_{c_{1} c_{2}}$ when $c_{2}=-0.76+0.1 i$ and $c_{1}$ takes different values in the neighborhood of $c_{2}$ can be connected, disconnected and totally disconnected.

In this section, according to Theorem 2.2, we verify graphically that the connectivity properties of the Julia sets of $Q_{c_{1} c_{2}}$ are transferred to the alternated Julia sets of $P_{c_{1} c_{2}}$.

\subsection{Connectivity zones}

The quartic polynomial $Q_{c_{1} c_{2}}$ has three critical points, $0, \sqrt{-c_{1}}$ and $-\sqrt{-c_{1}}$, but the orbits of $\pm \sqrt{-C_{1}}$ are the same, except in the initial point, because of the parity of $Q_{c_{1} c_{2}}$. According to the Fatou-Julia theorem, there are three possibilities about the orbits of the critical points of $Q_{c_{1} c_{2}}$ :

(i) The orbits of 0 and $\pm \sqrt{-c_{1}}$ are bounded. Then, the alternated Julia set $K_{c_{1} c_{2}}$ is connected.

(ii) The orbits of 0 and $\pm \sqrt{-c_{1}}$ are unbounded. Then, the alternated Julia set $K_{c_{1} c_{2}}$ is totally disconnected.

(iii) The orbit of 0 is bounded and the orbits of $\pm \sqrt{-C_{1}}$ are unbounded, or the orbit of 0 is unbounded and the orbits of $\pm \sqrt{-C_{1}}$ are bounded. Then, the alternated Julia set $K_{c_{1} c_{2}}$ is disconnected if the bounded critical orbits are periodic and it is totally disconnected if the bounded critical orbits are aperiodic. 
For a given parameter value $c_{2}$ it is possible to find, by means of a computer program, the connectivity zones of $c_{1}$ in the neighborhood of $c_{2}$ that origin connected, disconnected and totally disconnected alternated filled Julia sets $K_{c_{1} c_{2}}$. For example, in Fig. 2a the connectivity zones for $K_{c_{1} c_{2}}$ when $c_{2}=-0.76+0.1 i$, the real part of $c_{1}$ is in the interval $[-0.77,-0.75]$ and the imaginary part of $c_{1}$ is in the interval $[0.09,0.11]$, are drawn. Firstly, for each pixel of the figure the corresponding parameter value $c_{1}$ is determined. Secondly, the orbits of the critical points 0 and $\pm \sqrt{-C_{1}}$ of $Q_{c_{1} c_{2}}$ are tested to see if they are bounded or not. Finally, the color of the pixel is assigned (black if all the orbits are bounded, grey if there are both bounded and unbounded orbits, and white if all the orbits are unbounded).

Figure $2 \mathrm{~b}$ is a magnification of the square $\boldsymbol{a}$ of Fig. 2a with $c_{2}=-0.76+0.1 i$. The real part of $c_{1}$ is in the interval $[-0.766,-0.759]$ and the imaginary part of $c_{1}$ is in the interval $[0.099 i, 0.106 i]$. We have selected six representative points in the connectivity zones. The points $\mathrm{A}\left(c_{1}=-0.76+0.1 i\right)$ and $\mathrm{B}\left(c_{1}=-0.762+0.102 i\right)$, in the white zone, must correspond to totally disconnected alternated Julia sets. The points $\mathrm{C}\left(c_{1}=-0.7628+0.1028 i\right)$ and $\mathrm{E}\left(c_{1}=-0.764+0.104 i\right)$, in the grey zone, must correspond to disconnected alternated Julia sets. The point $\mathrm{D}\left(c_{1}=-0.763181+0.103171 i\right)$ is near the boundary of the grey/white zones. The point $\mathrm{F}\left(c_{1}=-0.765+0.105 i\right)$, in the black zone, must correspond to a connected alternated Julia set.

\subsection{Critical orbits}

Figure 3 shows in detail the orbits of the critical points 0 and $-\sqrt{-c_{1}}$ of $Q_{c_{1} c_{2}}$ corresponding to the points A, B, C, D, E and F of Fig. 2b (as we have said, the orbits of the two critical points $\pm \sqrt{-C_{1}}$ are the same except in the initial point). Note that the second point of the orbits of $\pm \sqrt{-C_{1}}$ is $c_{2}$.

In Figs. 3a and 3b, corresponding to points $\mathrm{A}$ and $\mathrm{B}$ of Fig. 2b, all the critical orbits are unbounded. 
In Fig. 3c, corresponding to point $\mathrm{C}$ of Fig. $2 \mathrm{~b}$, the orbits of the two critical points $\pm \sqrt{-c_{1}}$ are unbounded but the orbit of critical point 0 is period-15 periodic. Note that the point $\mathrm{C}$ is inside a disc of the disconnected zone (Fig. 2b), not in the main body of this zone.

The point $\mathrm{D}$ of Fig. $2 \mathrm{~b}$ is near the boundary between the grey and white zones (it is impossible, with a finite precision computer program, to determine a $c_{1}$ value in this boundary). In Fig. 3d, corresponding to point D, the orbits of the two critical points $\pm \sqrt{-c_{1}}$ are unbounded but the orbit of the critical point 0 is bounded. This critical orbit is non periodic. In the figure, the orbit contains 10,000 points and seems to be an aperiodic one. Taking into account the Banner-Hubbard conjecture, the corresponding alternated Julia set must be totally disconnected.

In Fig. 3e, corresponding to point $\mathrm{E}$ of Fig. 2b, the orbits of the two critical points $\pm \sqrt{-C_{1}}$ are unbounded, but the orbit of 0 is period- 1 periodic. Note that point $E$ is in the main body of the grey zone of Fig. $2 b$.

Finally, in Fig. 3f, corresponding to point F of Fig. 2b, all the critical orbits are period-1 periodic.

\subsection{Drawing alternated Julia sets}

In Fig. 4 three examples of alternated filled Julia sets $K_{c_{1} c_{2}}$ (on the left) and $K_{c_{2} c_{1}}$ (on the right) are shown when $c_{2}=-0.76+0.1 i$ and $c_{1}$ is in the neighborhood of $c_{2}$ (compare with Fig. 1). The critical points of polynomial $Q_{c_{1} c_{2}}$ are also shown. The totally disconnected alternated Julia sets of Fig. 4a correspond to point $\mathrm{B}\left(c_{1}=-0.762+0.102 i\right)$ of Fig. 2b, the disconnected alternated Julia sets of Fig. 4b correspond to point $\mathrm{C}\left(c_{1}=-0.7628+0.1028 i\right)$ of Fig. $2 \mathrm{~b}$, and the connected alternated Julia sets of Fig. 4c correspond to point $\mathrm{F}(-0.765+0.105 i)$ of Fig. $2 \mathrm{~b}$.

The alternated Julia sets $K_{c_{1} c_{2}}$ and $K_{c_{2} c_{1}}$ show a flashy graphical alternation. For example, in Fig. 4a, we can see the graphical alternation of the alternated Julia sets $K_{c_{1} c_{2}}: a b a^{\prime} b^{\prime} \ldots$ (on the left) and $K_{c_{2} c_{1}}: b a b^{\prime} a^{\prime} \ldots$ (on the right). 


\section{Conclusions}

In this paper we introduce the alternated Julia sets corresponding to the alternated iteration of two complex quadratic polynomials. We present, analytically and using computer graphics, which the alternated Julia sets can be connected, disconnected and totally disconnected, verifying the Fatou-Julia theorem in the case of complex polynomials of degree greater than two. Moreover, these alternated Julia sets exhibit graphical alternation.

\section{Acknowledgements}

This work was supported by the MEC, Spain, research grant SEG 2004-02418. We thank to Robert L. Devaney and Bodil Branner for their useful comments.

\section{References}

[1] J. Almeida, D. Peralta-Salas, M. Romera, Can two chaotic systems give rise to order? Physica D 200 (2005) 124-132.

[2] M. Romera, M. Small, M.-F. Danca, Deterministic and random synthesis of discrete chaos, Applied Mathematics and Computation 192 (2007) 283-297.

[3] M.-F. Danca, W. Tang, G. Chen, A switching scheme for synthesizing attractors of dissipative chaotic systems, submitted.

[4] P. Fatou, Sur les équations fonctionnelles, Bulletin de la Société Mathématique de France, 47 (1919) 161-271; 48 (1920) 33-94; 48 (1920) 208-314.

http://smf.emath.fr/Publications/Bulletin/

[5] G. Julia, Mémoire sur l'itération des fonctions rationnelles, Journal de Mathématiques Pures et Appliquées, 8 (1918) 47-245.

http://gallica.bnf.fr/ark:/12148/bpt6k1076109

[6] B. Branner, Puzzles and para-puzzles of quadratic and cubic polynomials. In Complex Dynamical System (R.L. Devaney Ed.), Proc. Symp. Appl. Math. 49, AMS (1994) 31-67.

[7] B. Branner and J.H. Hubbard, Iteration of cubic polynomials, Part II: Patterns and parapatterns, Acta Math. 169 (1992) 229-325.

[8] W. Qiu and Y. Yin, Proof of the Branner-Hubbard conjecture on Cantor Julia sets, preprint arXiv: math.DS/0608045 v1(2006).

[9] P. Blanchard, Disconnected Julia sets. In Chaotic Dynamics and Fractals (M. Barnsley, S. Demko Eds.) Academic Press (1986) pp. 181-201. 
[10] B. Branner and J.H. Hubbard, Iteration of cubic polynomials, Part I: The global topology of parameter space, Acta Math. 160 (1988) 143-206.

[11] P. Blanchard, R. Devaney and L. Keen, Complex dynamics and symbolic dynamics. In Symbolic Dynamics and its Applications (S.G. Williams Ed.) Proc. Symp. Appl. Math. 60 (2004) 37-60.

[12] R.L. Devaney and D.M. Look, A criterion for Sierpinski curve Julia sets, Topology Proceedings 30 (2006) 1-17.

[13] L. Carleson and T.W. Gamelin, Complex Dynamics, Springer-Verlag, New York, 1993, pp. 66-67.

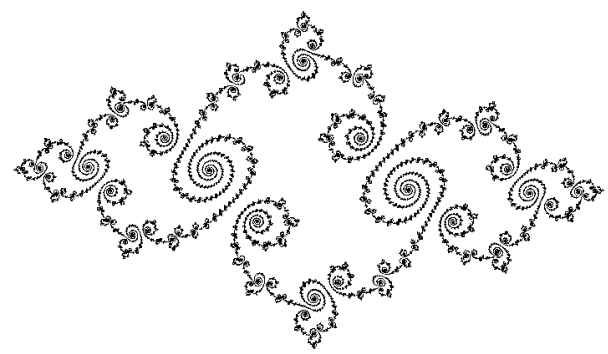

Figure 1.

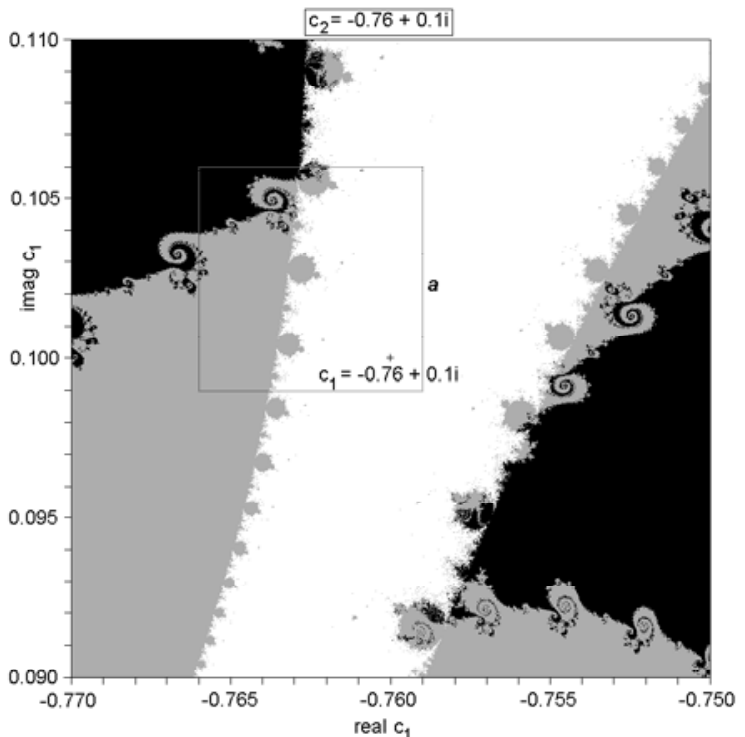

(a)

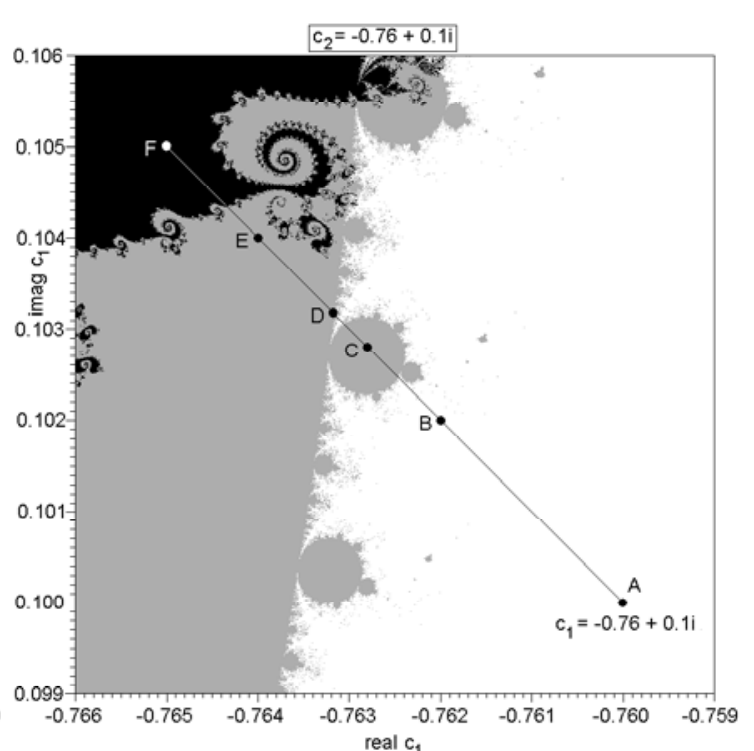

(b)

Figure 2 

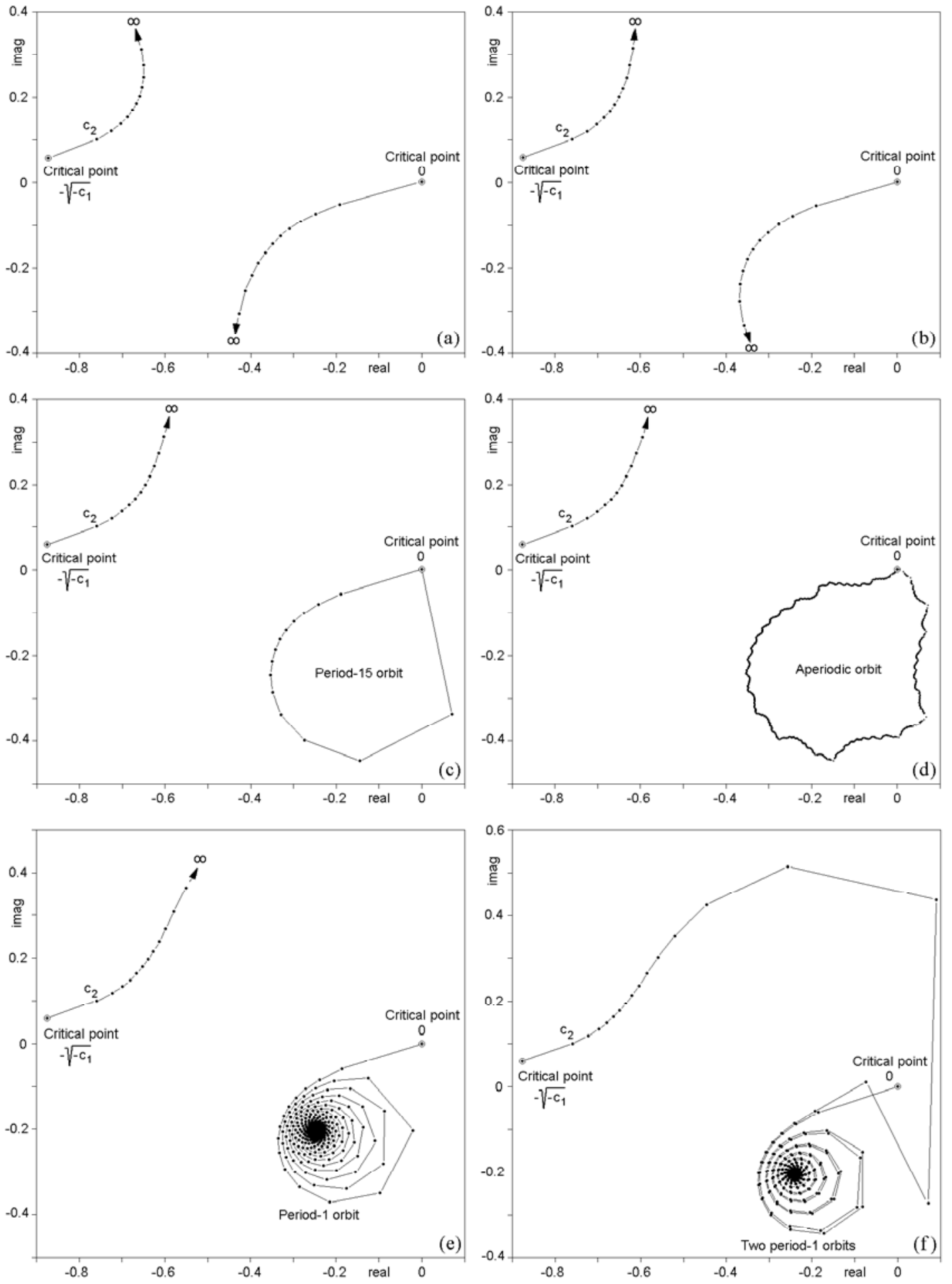

Figure 3. 

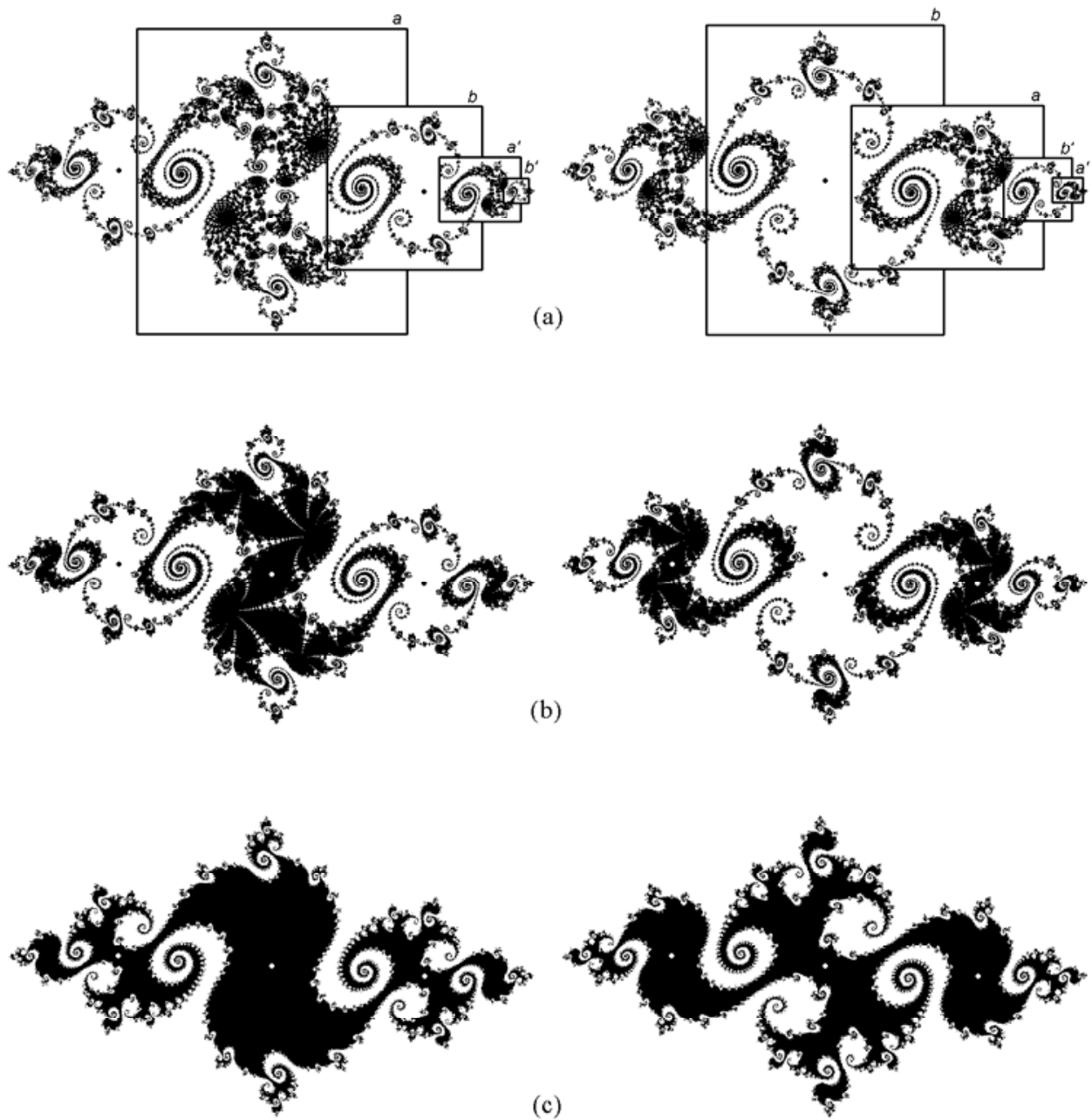

Figure 4

\section{Figure captions}

Fig. 1. Totally disconnected Julia set of $z_{n+1}=z_{n}^{2}+c$ when $c=-0.76+0.1 i$.

Fig. 2. Connectivity zones of the system $z_{n+1}=z_{n}^{2}+c_{1}$ ( $n$ even), $z_{n+1}=z_{n}^{2}+c_{2}$ ( $n$ odd) when $c_{2}=-0.76+0.1 i$ and $c_{1}$ is in the neighborhood of $c_{2}$. The black, grey and white zones correspond to connected, disconnected and totally disconnected alternated filled Julia sets $K_{c_{1}, c_{2}}$. a) The side of the window is 0.02 . b) Magnification of the square $\boldsymbol{a}$ of (a).

Fig. 3. Orbits of the critical points 0 and $-\sqrt{-C_{1}}$ of polynomial $Q_{c_{1} c_{2}}$ corresponding to the points A (a), B (b), C (c), D (d), E (e) and F (f) of Fig. 2 b. 
Fig. 4. Examples of alternated filled Julia sets $K_{c_{1} c_{2}}$ (on the left) and $K_{c_{2} c_{1}}$ (on the right) showing the critical points of polynomial $Q_{c_{1} c_{2}}$. (a) Totally disconnected Julia set (point B of Fig. 2b). (b) Disconnected Julia set (point C of Fig. 2b). (c) Connected Julia set (point F of Fig. 2b). 